\title{
IMPLANTAÇÃO DE UMA NOVA PROPOSTA PEDAGÓGICA PARA O ESTÁGIO SUPERVISIONADO EM FISIOTERAPIA NA ATENÇÃO BÁSICA: RELATO DE EXPERIÊNCIA
}

\author{
IMPLANTATION OF A NEW PEDAGOGICAL PROPOSAL FOR THE SUPERVISED INTERNSHIP IN \\ PHYSICAL THERAPY IN PRIMARY CARE: EXPERIENCE REPORT
}

\author{
Adriane Pires Batiston \\ Laís Alves de Souza Bonilha \\ Fernando Pierette Ferrari \\ Arthur de Almeida Medeiros \\ Clayton Duenha \\ Curso de Fisioterapia
}

Universidade Federal de Mato Grosso do Sul

\author{
Suely Grosseman \\ Departamento de Pediatria \\ Centro de Ciências da Saúde \\ Universidade Federal de Santa Catarina
}

Roberto Zonato Esteves

Curso de Medicina

Universidade Estadual de Maringá

\author{
Contato \\ Adriane Pires Batiston \\ Cidade Universitária $s / n$ \\ Campo Grande-MS \\ CEP: 79070-900 \\ E-mail: abatiston@gmail.com
}

\section{RESUMO}

O estágio de Fisioterapia na atenção básica, do curso de Fisioterapia da Universidade Federal de Mato Grosso do Sul, realizado nas Unidades Básicas de Saúde da Família, sempre se configurou como um desafio pela inexistência do profissional fisioterapeuta na Estratégia Saúde da Família em Campo Grande/MS e pelo modelo de trabalho desenvolvido pelas equipes que possuíam uma visão limitada das possibilidades de atuação do fisioterapeuta. O processo de trabalho na Estratégia Saúde da Família pode ser transformado por estratégias que possibilitem a experiência do aprendizado e do trabalho em equipe para todos os atores envolvidos, aproximando o mundo da formação do mundo do trabalho. Assim, o objetivo deste artigo foi relatar a experiência da implantação de uma nova proposta pedagógica para o Estágio Supervisionado em Fisioterapia na Atenção Básica. A nova proposta pedagógica foi construída coletivamente pelos docentes e fisioterapeutas da universidade e pelas equipes de Saúde da Família e foi apoiada em uma nova lógica do trabalho dos estagiários, estando estes inseridos em ações de educação, cuidado, gestão e participação social, tendo uma equipe de referência dentro da qual realizou todas as atividades previstas para o desenvolvimento de competências esperadas para sua formação nesse nível de atenção. A partir da avaliação dos primeiros estudantes participantes da nova proposta implantada, identificaram-se impactos positivos da experiência, com destaque para o sentimento de pertencimento do aluno à equipe de Saúde da Família à qual estava vinculado, avançando na prática interprofissional e na integração do estudante com pacientes e familiares, propiciando cuidado mais resolutivo; e contribuindo para a mudança da percepção da equipe sobre as possibilidades de atuação do fisioterapeuta na atenção básica.

Palavras-chave: Fisioterapia. Atenção Primária à Saúde. Educação Superior. 


\section{ABSTRACT}

The internship in Physical Therapy within Primary Care of the Physical Therapy course of the Federal University of Mato Grosso do Sul, conducted in the Family Health Basic Units, has always been a challenge due to the lack of physical therapists in the Family Health Strategy in Campo Grande/MS, and also because of the work model developed by teams that had a limited view of the possibilities of action of physical therapists. The work process in the Family Health Strategy can be transformed by strategies that allow the experience of learning and of teamwork for all the actors involved, bringing the world of training closer to the world of work. Thus, the objective of this article is to report the experience of the implementation of a new pedagogical proposal for the Supervised Internship in Physical Therapy in Primary Care. The new pedagogical proposal was built collectively by the faculty and physical therapists of the university and by the Family Health teams, and was supported by a new logic of interns' work, being inserted in actions of education, care, management and social participation, with a reference team within which it carried out all the planned activities for the development of the expected competencies for its qualification in this level of care. From the evaluation of the first students participating in the new implemented proposal, positive experiences were identified, highlighting the student's sense of belonging to the Family Health team to which they were linked, advancing in interprofessional practice and student integration with patients and family members, providing more resolute care; and contributing to the change in the perception of the team about the physical therapist's possibilities in Primary Care.

Keywords: Physical Therapy Specialty. Primary Health Care. Education, Higher.

\section{CONTEXTUALIZAC̣ÃO}

$\mathrm{O}$ fisioterapeuta brasileiro, em seus primórdios conhecido como paramédico, passou à condição de técnico em Fisioterapia em 1963, ainda como auxiliar dos médicos; e apenas em 1969, foi reconhecido como profissional de nível superior pelo Decreto 938/691,2. Assim, a Fisioterapia, como outras profissões da área da saúde, originou-se de uma subdivisão da Medicina, que, como primeira profissão da área da saúde, passou a especializar-se e ramificar-se em outras profissões.

Em suas quatro primeiras décadas, o fisioterapeuta ganhou reconhecimento social e criou sua identidade profissional fortemente relacionada com ações de recuperação funcional de agravos físicos ${ }^{3}$. No campo da reabilitação, desenvolveu novas tecnologias, avançou na produção de evidências científicas e consolidou sua atuação como membro de equipes de saúde nos níveis de atenção secundário e terciário ${ }^{3,4}$.

A Fisioterapia, entretanto, não acompanhou a evolução das políticas de saúde, especialmente após a criação do Sistema Único de Saúde (SUS) e reorientação do modelo assistencial brasileiro, que instituiu a Atenção Básica $(A B)$ como estratégia prioritária para os cuidados em saúde. $A A B$ deve ser a porta de entrada preferencial dos usuários e centro de comunicação com toda a Rede de Atenção à Saúde (RAS), devendo orientar-se pelos princípios da universalidade, da acessibilidade, do vínculo, da continuidade do cuidado, da integralidade da atenção, da responsabilização, da humanização, da equidade e da participação social ${ }^{5}$.
A Estratégia Saúde da Família (ESF) foi implantada com vistas a potencializar a reorganização da $A B$, que se fundamenta na vigilância à saúde e possui a família como foco principal de sua atenção, a partir do seu ambiente físico e social. Uma das características da ESF é o trabalho exercido por uma equipe multiprofissional formada em sua constituição básica por um médico, um enfermeiro, um auxiliar ou técnico de enfermagem e seis agentes comunitários de saúde. Quando ampliada, essa equipe inclui ainda um dentista, um auxiliar de consultório dentário e um técnico em higiene dental ${ }^{5}$. O fisioterapeuta não está inserido na equipe básica da ESF. Entretanto, ele pode integrar as equipes ampliadas da ESF ou compor, juntamente com outros profissionais, o Núcleo de Apoio à Saúde da Família (NASF).

A oferta de cuidado integral, buscando responder de forma mais efetiva às necessidades de saúde da coletividade, pressupõe maior acesso da população a ações de promoção, prevenção, recuperação e reabilitação6. Isto requer uma atuação integrada e colaborativa de uma gama de profissionais da saúde para além do médico; entre eles, o fisioterapeuta. Assim, é imprescindível que os profissionais experimentem o trabalho interprofissional desde sua formação na graduação, estando assim preparados para o mundo do trabalho 6 .

$A$ inserção do fisioterapeuta na $A B$ e em especial na ESF, embora venha adquirindo crescente importância nos serviços de saúde, tem ocorrido muito timidamente, o que sugere que o acesso à atenção fisioterapêutica está bem aquém do necessário para o cuidado integral à saúde das coletividades ${ }^{7}$. Atu- 
ar como membro das equipes de Saúde da Família (eSF), portanto, requer do fisioterapeuta a utilização de tecnologias leves e leve-duras e o desenvolvimento de competências para um fazer reflexivo, dotado de novos sentidos e coerentes com a proposta da ESF8.

A presença do fisioterapeuta na ESF, além da contribuição para o cuidado integral, poderia em muito auxiliar a equipe no enfrentamento das doenças crônicas não transmissíveis, considerando-se o perfil epidemiológico contemporâneo, reduzindo os riscos de comprometimentos funcionais por meio de ações voltadas para indivíduos e grupos, no sentido de meIhorar ou preservar o desempenho físico-funcional com impactos diretos na vida das pessoas ${ }^{9}$.

No que tange à formação do fisioterapeuta, a publicação das Diretrizes Curriculares Nacionais para o Curso de Graduação em Fisioterapia (DCN/ Fisio) ${ }^{10}$, em 2002, mobilizou escolas e organizações sociais a fim de revisarem seus projetos pedagógicos, comprometendo-se com a formação de profissionais para atuarem no sistema de saúde vigente no País, nos três níveis de atenção, inclusive na $A B$.

Passados 14 anos da implantação das DCN/Fisio e da construção de conhecimentos significativos sobre a inserção do fisioterapeuta na $A B$, todavia, a incorporação desse profissional na ESF esbarra em diversos fatores que acabam por criar um ciclo crítico: o SUS necessita de profissionais fisioterapeutas dotados de novas competências para atuar na AB. Essa necessidade desencadeou mudanças nas escolas, que passaram a incluir disciplinas e estágios em atenção básica em seus currículos de graduação. Os estudantes, porém, nem sempre conseguem vivenciar a realidade de atuação do fisioterapeuta na $A B$, uma vez que esse profissional não está inserido nesse nível com a intensidade necessária. Isso desmotiva os estudantes a idealizarem sua atuação profissional nesse nível de atenção, interferindo na qualidade dos profissionais que acabam sendo absorvidos pelo sistema de saúde, muitas vezes, sem o domínio de competências para atender às expectativas da equipe, dos gestores e, principalmente, da população.

\section{Identificação do Problema}

O curso de Fisioterapia da Universidade Federal de Mato Grosso do Sul (UFMS) foi criado em 2008 e caracteriza-se como o único curso de Fisioterapia de caráter público em Mato Grosso do Sul. Desde a sua criação, o curso apostou em um Projeto Pedagógico comprometido com princípios e diretrizes do SUS e com as necessidades da população brasileira, que ainda enfrenta dificuldades de acesso ao profissional fisioterapeuta, especialmente na AB. Ele estrutura-se a partir do quadrilátero da formação proposto por Ceccim e Feuerwerker $(2004)^{9}$ e busca formar profissionais que também pro- movam a saúde e previnam doenças musculoesqueléticas, crônico-degenerativas e outras decorrentes do aumento da expectativa de vida, além de atuarem em práticas já fortemente instituídas do fisioterapeuta nos níveis de atenção secundário e terciário, desenvolvidas prioritariamente em ambulatórios, clínicas e hospitais.

Em 2013, iniciaram-se os estágios obrigatórios do curso de fisioterapia da UFMS, momento em que se firmou um convênio com a Secretaria Municipal de Saúde de Campo Grande para a inserção dos alunos do $9^{\circ}$ e $10^{\circ}$ semestres na ESF. A nova proposta constituiu-se como desafiadora para todos os atores envolvidos, pois a chegada de estudantes provenientes de um curso, cuja categoria profissional não estava inserida nesse nível de atenção, certamente provocaria mudanças no processo de trabalho dessa equipe e no cuidado das pessoas atendidas. Diante disso, verificou-se a necessidade de investigação acerca do conhecimento dos profissionais da eSF sobre a atuação do fisioterapeuta no âmbito da $A B$. A pesquisa realizada demonstrou que, na percepção da maioria dos profissionais, o fisioterapeuta atuava com pessoas portadoras de doenças já instaladas, com foco na reabilitação e cura, em detrimento de ações de promoção de saúde e prevenção de agravos.

A falta de conhecimento dos profissionais sobre a atuação do fisioterapeuta na $A B$ parece refletir também a visão dos gestores e da própria população que, culturalmente, também relacionam o fisioterapeuta com práticas essencialmente reabilitadoras. Esse fato representava uma barreira para o desenvolvimento de ações de promoção da saúde e prevenção de agravos na população. Por outro lado, essas práticas são extremamente importantes para o cuidado integral e mudança na condição de saúde das pessoas.

Passados dois anos do início das atividades dos estagiários de Fisioterapia na Unidade Básica de Saúde da Família Macaúbas, onde estão alocadas três eSF, percebeu-se que a mudança da visão da equipe de saúde e da comunidade sobre a atuação do fisioterapeuta vinha se ampliando em velocidade e qualidade aquém do esperado, ou seja, os encaminhamentos da equipe e a procura espontânea da comunidade eram, em grande parte, para atenção fisioterapêutica a pessoas já acometidas por agravos e comprometimentos funcionais significativos e que, portanto, não se beneficiariam de atenção em nível primário.

Mesmo estando diariamente na Unidade Básica de Saúde da Família, os estudantes de fisioterapia, sob supervisão de docente fisioterapeuta, desenvolviam suas ações dissociadas das atividades das eSF. Como consequência, na avaliação de sua experiência na ESF, eles descreviam dificuldade de interação com a equipe, além de muitas vezes sentirem-se solitários e impotentes para resolução dos problemas de indivíduos e famílias sob seus cuidados. 
Os estudantes também referiam dificuldades de comunicação com a equipe de saúde e desconheciam algumas ações que estavam em desenvolvimento, nas quais poderiam contribuir para melhorar a qualidade da atenção ofertada. Além disso, o desconhecimento das equipes sobre as possibilidades de atuação do fisioterapeuta em ações com objetivos de promoção da saúde e prevenção de agravos aumentava a frequência de encaminhamentos de "pacientes" restritos ao leito ou com sérios comprometimentos físico-funcionais, e, paralelamente, os profissionais da equipe não se envolviam com as ações propostas pela fisioterapia na unidade. Diante disso, a partir de reflexões suscitadas, foi desenvolvida uma nova proposta pedagógica para o estágio na $A B$, buscando-se uma maior aproximação entre o processo profissionalizante da formação e o mundo do trabalho, valorizando-se todo o potencial de atuação dos estudantes de Fisioterapia em ações de promoção, prevenção, reabilitação e tratamento nesse nível de atenção.

Dessa forma, o objetivo deste artigo é relatar a experiência da implantação de uma nova proposta pedagógica para o Estágio Supervisionado em Fisioterapia na $\mathrm{AB}$.

\section{DESCRIÇÃO DA EXPERIÊNCIA}

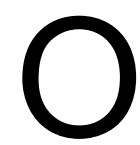

presente trabalho apresenta o relato da experiência, em que, por meio de abordagem qualitativa, foram coletadas as percepções dos quatro alunos envolvidos na implantação inicial da proposta pedagógica, objeto deste relato. A análise dos dados foi realizada por análise temática de conteúdo. Os temas emergentes estão ressaltados em negrito.

\section{O processo de elaboração e implantação do estágio obrigatório na $A B$}

A partir dos objetivos do estágio obrigatório de Fisioterapia na AB (quadro 1), a implantação da proposta desenvolveu-se em quatro fases distintas:

\section{Quadro 1. Objetivos do Estágio em Atenção Básica (Estágio Obrigatório em Território de Prática I) do curso de fisioterapia da Universidade Federal de Mato Grosso do Sul}

\section{Objetivos do Estágio de Fisioterapia em Atenção Básica}

1. Desenvolver competências para a execução de ações em equipe voltadas para a saúde da família, do adulto, do idoso, da mulher, da criança e do adolescente.

2. Desenvolver competências para o diagnóstico físico funcional de adultos, idosos, crianças e adolescentes em diferentes condições clínicas na Unidade Básica de Saúde da Família (UBSF) e domicílios.

3. Desenvolver competências para a tomada de decisão na seleção e aplicação de recursos terapêuticos.

4. Desenvolver competências para a identificação e redução de riscos, a prevenção e minimização de agravos considerando-se os determinantes de saúde e os indicadores epidemiológicos e demográficos.

5. Desenvolver competências para proposição e execução de projeto terapêutico singular em equipe multiprofissional.

6. Desenvolver competências para a participação e condução de ações de educação permanente nas equipes.

7. Desenvolver competências para a participação e condução de ações de educação em saúde na comunidade.

8. Proporcionar a participação e reconhecimento do controle social na atenção primária.

9. Proporcionar a vivência no território de saúde identificando particularidades e potencialidades dos equipamentos sociais e dos serviços de saúde.

10. Identificar a importância e a necessidade de criação de linhas de cuidado e modos de construí-las em rede.

11. Desenvolver conhecimento sobre as ferramentas de gestão e sobre redes de saúde. 


\section{Fase 1}

A primeira fase consistiu na reunião com o grupo de professores fisioterapeutas que realizam a supervisão direta dos estudantes dos $9^{\circ} \mathrm{e} 10^{\circ}$ semestres de Fisioterapia em duas Unidades Básicas de Saúde da Família, juntamente com os fisioterapeutas do curso, que também desenvolvem a supervisão compartiIhada no território. Essa reunião teve como objetivo a avaliação do estágio, para identificar como ele estava se desenvolvendo, as fragilidades e potencialidades percebidas e as estratégias de mudanças sugeridas para melhorar o processo pedagógico.

Identificou-se que a pouca integração dos alunos e professores da UFMS com as equipes de saúde era a fragilidade principal, uma vez que esta desencadeava as demais dificuldades. Nesse aspecto, foram identificados a falta de comunicação, o desconhecimento sobre o papel da fisioterapia na $A B$, a desarticulação das ações serviço-universidade, a inexistência de trabalho em equipe com a inclusão dos estudantes e o perfil de pacientes encaminhados para a fisioterapia. No que diz respeito ao processo ensino-aprendizagem, os professores identificaram que os instrumentos avaliativos não eram satisfatórios para a avaliação das competências motoras, afetivas e cognitivas, sendo o conhecimento mais valorizado em detrimento das habilidades e atitudes. A partir dessa avaliação, o grupo traçou as seguintes estratégias:

1. O grupo de estagiários que anteriormente atendiam a Unidade de Saúde agora estaria individualmente inserido em uma das eSF, participando de todas as ações previstas para essa equipe, portanto, cada estudante teria um tutor que acompanharia o desenvolvimento e, com isso, também se vincularia à equipe de referência.

2. Cada aluno deveria cumprir um rol de atividades planejadas com seu tutor, a fim de alcançar os objetivos do estágio, sendo elas: territorialização (diagnóstico situacional de sua área a partir de reconhecimento do território) e participação nas reuniões da equipe; visitas domiciliares com os agentes comunitários de saúde para avaliar os usuários com indicação para fisioterapia e eleger dois pacientes para atendimento domiciliar individual e identificar famílias e indivíduos que necessitassem de orientações e acompanhamento com vistas à promoção e prevenção de agravos; participação de, no mínimo, uma visita domiciliar compartilhada com enfermeiro, médico e agentes comunitário de saúde da equipe; participação em, no mínimo, uma consulta compartiIhada; participar das atividades educativas da equipe inclusive responsabilizando-se por ações; apoiar a equipe em ações pontuais dos programas de saúde; implementar a partir dos indicadores, um grupo terapêutico a ser desenvolvido com grupos vulneráveis; desenvolver um plano de cuidado compartilhado (PCC) para cada um dos pacientes, apresentando a avaliação do paciente e o plano de cuidado; participar de, no mínimo, uma reunião do Conselho Municipal de Saúde, uma reunião do Fórum de Trabalhadores da Saúde e uma reunião do Conselho local de saúde, desenvolvendo conhecimentos sobre o controle social; e realizar uma avaliação teórica.

3. Desenvolvimento e utilização de novos instrumentos de avaliação do estudante. Instrumento 1 para avaliação diária dos estudantes contendo uma lista de competências esperadas durante o estágio, tais como: a) comunicação e relação com pacientes, familiares, equipe de saúde e outros estudantes; b) responsabilidade com documentos e equipamentos; c) competência para o trabalho em equipe; d) criatividade; e) iniciativa; f) liderança; g) habilidades de gestão; h) conhecimento técnico crescente compatível com a complexidade de seus pacientes e suas necessidades; i) habilidade de planejar e pactuar suas atividades com paciente e equipe; j) visão ampliada da saúde e proposição de ações que favoreçam o cuidado integral, entre outros. Instrumento 2 para avaliação do PCC: sendo considerados critérios como apresentação dos dados do usuário de forma completa, conforme as especificidades deste, descrição dos agravos de forma completa, narrativa analítica do itinerário terapêutico na rede de atenção à saúde, risco familiar, elaboração adequada da avaliação, diagnóstico fisioterapêutico, objetivos e condutas, considerando-se o conceito ampliado de saúde, o trabalho em equipe e a intersetorialidade. Instrumento 3 para avaliação das fichas de avaliação e evolução dos indivíduos atendidos. Instrumento 4 para avaliação de seminários contendo itens como a preparação, utilização de melhores evidências, relação teoria-prática, complexidade de conhecimento compatível com o momento do estágio e necessidades dos pacientes, e incentivo a participação dos colegas, além da avaliação teórica. Todas as avaliações apoiadas nos instrumentos descritos eram oportunamente discutidas pelo tutor e estudante de forma continuada, com feedback mais frequente possibilitando ao estudante participar ativamente de seu processo pedagógico, conhecendo suas fragilidades e potencialidades.

4. Criação de um canal de comunicação mais eficiente entre os professores e supervisores do estágio, minimizando as falhas de comunicação e sanando possíveis lacunas de forma mais rápida e eficiente. 


\section{Fase 2}

A fase 2 consistiu em reuniões com as eSF, para apresentar a nova proposta detalhada anteriormente na fase 1 e discutir a sua operacionalização. Nessa oportunidade, estavam presentes todos os profissionais das três equipes da Unidade (médicos, enfermeiros, técnicos e auxiliares, agentes comunitários de saúde e odontólogos); participaram ainda a gerente, a assistente social e os profissionais que atuam na recepção da unidade. Foram apresentadas para as equipes a necessidade de alinhamento dos objetivos pedagógicos com as necessidades da população e foram levantadas as expectativas da equipe quanto à atuação dos estudantes. Foi reforçado o papel do fisioterapeuta em ações de promoção, prevenção de agravos, educação em saúde, gestão e controle social, além das já reconhecidas ações de reabilitação e tratamento. Foi também bastante discutida com a equipe a importância da integração ensino-serviço bem como os seus objetivos, destacando-se a necessidade de a equipe acolher os estudantes e professores de fisioterapia, garantindo a inserção deles em todas as atividades da equipe, desde as reuniões de planejamento, visitas e consultas compartilhadas, ações educativas, ações programáticas e integração com os estudantes de outras profissões atuantes na mesma equipe. A proposta foi bem aceita, e, nesse momento, cada enfermeira coordenadora de equipe foi informada sobre o aluno que integraria sua equipe e o tutor responsável, lembrando que a supervisão é realizada de forma direta por um professor e um profissional da UFMS, ambos fisioterapeutas, não havendo o fisioterapeuta do serviço propriamente dito.

\section{Fase 3}

Apresentação da proposta aos estudantes. Após serem explicadas cada uma das atividades a serem desenvolvidas e apresentados os instrumentos de avaliação, os estudantes identificaram seus tutores e puderam iniciar a elaboração de seu plano de trabalho. Eles também receberam informações sobre as equipes nas quais seriam inseridos. Nessa fase, a nova proposta foi operacionalizada, sendo que os estudantes permaneceram nesse estágio por 9 semanas, com carga horária semanal de 30 horas, totalizando 270 horas.

\section{Fase 4}

Como os estudantes eram os atores centrais da experiência, esta foi avaliada pelos quatro estudantes que desenvolveram as atividades propostas. Para tanto, realizou-se uma roda de conversa, na qual eles avaliaram seu estágio, destacando as competências desenvolvidas, os pontos positivos e negativos e a contribuição do estágio em sua formação profissional.

\section{RESULTADOS}

A partir dos relatos dos estudantes do $9^{\circ}$ semestre do curso de Fisioterapia, que iniciaram seu estágio na $A B$, foi possível identificar os impactos da experiência de inserção dos estudantes nas diferentes eSF da Unidade Básica de Saúde da Família Macaúbas, apresentados a seguir.

\section{O estudante de fisioterapia como membro da equipe de Saúde da Família}

A experiência aqui descrita traz como elemento de inovação a inserção dos estagiários de Fisioterapia nas eSF, participando de todas as atividades previstas pela equipe na qual estavam inseridos, rompendo com a lógica muito comum na formação de fisioterapeutas, na qual os estagiários são supervisionados pelo professor fisioterapeuta, o que reduz as oportunidades de convivência e trocas com outros profissionais. Essa aproximação conferiu aos estudantes o sentimento de maior pertencimento àquela equipe, da mesma forma que a equipe acolheu os estagiários, integrando-os às suas atividades.

\section{O trabalho em equipe e o cuidado integral}

Os estagiários reconheceram os benefícios dessa experiência para sua formação e para a resolutividade dos problemas apresentados pelos indivíduos e grupos, ressaltando a importância do cuidado integral e a necessidade de uma equipe que trabalhe na lógica da interprofissionalidade.

\section{O fazer do fisioterapeuta da ESF: novas percepções}

Uma das inquietações que motivou essa experiência foi o desconhecimento das possibilidades de atuação do fisioterapeuta na ESF, com ações de promoção de saúde e prevenção de doenças, além das ações já reconhecidas visando à recuperação e à reabilitação. A despeito da presença dos estagiários na Unidade de Saúde, a equipe não compreendia que a contribuição do fisioterapeuta nesse nível de atenção poderia se dar com pessoas saudáveis ou portadoras de condições crônicas como a diabetes mellitus, a hipertensão arterial, alterações posturais, gestantes, entre outras. Mesmo com uma participação ativa nas reuniões, o encaminhamento de pacientes para a atenção fisioterapêutica era massivamente de pacientes restritos ao leito, já acometidos por condições graves e que, portanto, necessitavam do fisioterapeuta reabilitador.

A partir dessa nova experiência, percebeu-se a mudança nesse cenário: as constantes reuniões e ações conjuntas possibilitou a compreensão e aderência da equipe a novas propostas do papel do fisioterapeuta. 


\section{IMPACTOS}

$\mathrm{R}$ efletindo sobre o processo de mudança e a percepção dos estudantes que estagiaram na AB após sua implementação, pode-se perceber que os cursos de graduação em Fisioterapia devem oportunizar a inserção dos estudantes nesse nível de atenção desde o início do curso. A integração ensino-serviço-comunidade com a proposta de promover a saúde e prevenir seus agravos, além da reabilitação, rompe o paradigma de que a formação e atuação do fisioterapeuta seja realizada apenas em hospitais e clínicas, mais focada em competências motoras em detrimento das competências afetivas e cognitivas. $\mathrm{O}$ estudante precisa vivenciar sua possibilidade de fazer e construir o cuidado na $A B$, com todos os atributos que a diferenciam de outros níveis de atenção.

O fisioterapeuta tem sua origem fortemente ligada a ações de reabilitação e recuperação ${ }^{10,14,15}$, há de se reconhecer os avanços observados a partir do conceito ampliado de saúde, das mudanças demográficas, sociais e epidemiológicas, da reorganização do SUS e das DCN/Fisio ${ }^{16}$, atualmente em processo de reformulação, que orientam a formação de um profissional generalista capaz de atuar em todos os níveis de atenção à saúde, mas ainda há um longo caminho a trilhar na direção da ampliação do acesso da população a fisioterapia, especialmente na ESF.

Sabe-se que para que o fisioterapeuta desenvolva idealmente um trabalho na ESF, as instituições formadoras precisam comprometer-se com o desenvolvimento de competências essenciais nesse nível de atenção, ampliando as ferramentas da valise tecnológica desses futuros profissionais ${ }^{17}$. Pode-se dizer que a inserção do fisioterapeuta na $A B$ é uma construção que tem seus alicerces na formação qualificada de modo a habilitá-los para atuar nesse nível ${ }^{14}$.

Apesar de a inserção de alunos de Fisioterapia na $A B$ ser um grande desafio para a maioria das Instituições de Ensino Superior e dos serviços de saú$\mathrm{de}^{10}$, pode-se constatar que ela é viável. O desafio ocorre porque o Fisioterapeuta está pouco inserido nas eSF. No caso do município de Campo Grande, não há nenhum fisioterapeuta atuando diretamente nas eSF. Os profissionais fisioterapeutas que atuam nos NASF e apoiam as equipes possuem ferramentas específicas e competências distintas das requeridas na $\mathrm{ESF}^{12,13}$.

Diante dessa realidade, estratégias de integração dos estagiários de Fisioterapia com profissionais e estudantes de outras profissões nos cenários de prática devem ser implementadas de forma permanente, evitando que os estudantes se sintam descontextualizados do trabalho desenvolvido pela equipe, bem como favorecendo o desenvolvimento de sentidos e sentimentos sobre sua própria prática, como contribuinte do cuidado integral na ESF.

A avaliação produzida pelos estagiários sobre a nova proposta pedagógica demonstra sua percepção sobre a importância do trabalho em equipe para o cuidado integral. Esse reconhecimento é um passo importante para profissionais em formação, uma vez que provoca reflexões sobre a necessidade de diferentes olhares e práticas para atender às singularidades das pessoas e famílias na ESF. O trabalho em equipe é condição sem a qual não se oferta o cuidado integral, assim, trabalhar com e para o outro pressupõe a possibilidade da prática de um profissional se reconstruir na prática do outro ${ }^{13}$ e na vivência da realidade na qual a vida das pessoas acontece. Esse trabalho em equipe na eSF requer a integração dos diferentes profissionais, respeitando-se seus núcleos de saberes, sem perder de vista o campo da saúde, no qual os profissionais se apoiam, inovam, criam e, assim, ampliam as possibilidades de práticas resolutivas com qualidade, com atenção aos princípios e atributos da $A B$, especialmente da ESF?

\section{CONSIDERAC̣ÕES FINAIS}

A implementação da nova proposta pedagógica para os estagiários do curso de Fisioterapia da UFMS na ESF possibilitou maior integração com a equipe, favorecendo o alinhamento das ações com vistas a atender às necessidades da população, do serviço e da universidade.

Um dos pontos que merece destaque é a possibilidade de avanços na direção do cuidado integral, pautando-se no conceito ampliado de saúde, da visão do indivíduo de forma holística e do trabalho em equipe.

Essa experiência possibilitou a mudança da percepção da equipe sobre as possibilidades de atuação do fisioterapeuta na ESF, reconhecendo a importância desse profissional no desenvolvimento de ações de promoção da saúde e prevenção de agravos, além das ações de reabilitação e recuperação já estabelecidas no senso comum.

Essa integração amplia o interesse dos estudantes para uma futura atuação na $A B$, favorecendo a formação de profissionais cada vez mais preparados para atuar de forma resolutiva e qualificada nesse nível de atenção. Dessa forma, amplia-se seu potencial de promover a saúde da população. 


\section{Referências}

1. Salmória JG, Camargo WA. Uma Aproximação dos Signos - Fisioterapia e Saúde - aos Aspectos Humanos e Sociais. Saude soc. 2008; 17(1):73-84.

2. Brasil. Decreto-lei 938 de 13 de outubro de 1969. Provê sobre as profissões de fisioterapeuta e terapeuta ocupacional e dá outras providências. Diário Oficial da União, Brasília, n.197, seção 1, p.3658. 16 out. 1969.

3. Rocha VM, Câmara AMCS, Ferrari F, Batiston AP. Movimento Associativo e Educativo da Fisioterapia - Abenfisio e o Fórum Nacional de Educação das Profissões da Área da Saúde - Fnepas. Cad.FNEPAS.2012; v. 2:18-24.

4. Dean E, Al-Obaidi S, De Andrade, Gosselink R, Umerah G, Al-Abdelwahab S et al. The First Physical Therapy Summit on Global Health: implications and recommendations for the 21st century. Physiother Theory Pract. 2011;27(8):531-47.

5. Brasil. Ministério da Saúde. Departamento de Atenção Básica. Política Nacional de Atenção Básica. Brasília, 2012. Disponível em: http://189.28.128.100/dab/docs/publicacoes/geral/pnab.pdf. Acesso em 20 de março de 2015.

6. Peduzzi M. O SUS é interprofissional. Interface (Botucatu). 2017; 20(56):199-201.

7. Trelha CS, Silva D W, lida LM, Fortes MH, Mendes TS. O fisioterapeuta no programa de saúde da família em Londrina (PR), Rev Esp Saude 2007; 8(2):20-25.

8. Rezende M, Moreira MR, Amâncio Filho A, Tavares MFL. A equipe multiprofissional da 'Saúde da Família': uma reflexão sobre o papel do fisioterapeuta. Ciênc. saúde coletiva. 2009; 14(Suppl 1):1403-1410.

9. Santos MLM, Medeiros AA, Batiston AP, Pontes ERJ, Ferrari FP, Fernandes JM, Rios TA, Muzili NA, Sanches VS. Competências e atribuições do fisioterapeuta na Atenção Primária à Saúde. Fisioter Bras 2014; 15(1):69-76.

10. Brasil. Ministério da Educação. Resolução n. 4. aprovada em 19 de fevereiro de 2002. Institui Diretrizes Curriculares Nacionais do Curso de Graduação em Fisioterapia. Diário Oficial da União, de 4 de março de 2002. Seção 1, p. 11.

11. Ceccim RB, Feuerwerker LCM. O quadrilátero da formação para a área da saúde: ensino, gestão, atenção e controle social. Physis: Rev. de Saúde Coletiva. 2004; 14(1):41-65.

12. Novais BKLO, Brito GEG. Percepções sobre o trabalho do fisioterapeuta na atenção primária. Rev. APS. 2011; 14(4):424-34.

13. Fernandes JM, Rios TA, Sanches VS, Santos MLM. NASF's tools and practices in health of physical therapists. Fisioter. mov. 2016; 29(4):741-750.

14. Braghini CC, Ferretti F, Ferraz L. Physiotherapist's role in the NASF: perception of coordinators and staff. Fisioter. mov. 2016; 29(4):767-776.

15. Araújo MBS, Rocha PM. Trabalho em equipe: um desafio para a consolidação da estratégia de saúde da família. Ciênc. saúde coletiva. 2007; 12(2):455-464.

16. Formiga NFB, Ribeiro KSQS. Inserção do fisioterapeuta na atenção básica: uma analogia entre experiências acadêmicas e a proposta dos Núcleos de Apoio à Saúde da Família (NASF). R Bras Ci Saúde. 2012; 16(2):113-122.

17. Bispo Júnior JP. Fisioterapia e saúde coletiva: desafios e novas responsabilidades profissionais. Cien Saude Colet 2010; 15(Supl 1):1627-36.

18. Rocha VM, Caldas MAJ, Araújo FEO, Ragasson CAP, Santos MLS, Batiston AP. As diretrizes curriculares e as mudanças na formação de profissionais fisioterapeutas (ABENFISIO). Fisioterapia Brasil 2010; (Supl 1):4-8.

19. Neves LMT, Aciole GG. Desafios da integralidade: revisitando as concepções sobre o papel do fisioterapeuta na equipe de Saúde da Família. Interface. 2011; $15(3$ ):551-564. 